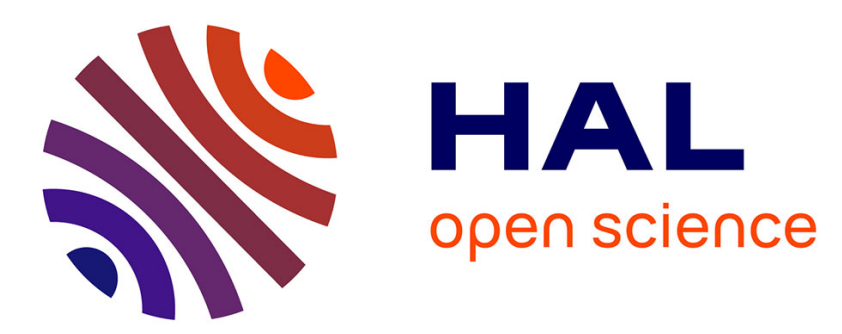

\title{
Une formulation variationnelle du problème de contact avec frottement de Coulomb
}

\author{
Anh Le Van, Tai H.T. Nguyen
}

\section{To cite this version:}

Anh Le Van, Tai H.T. Nguyen. Une formulation variationnelle du problème de contact avec frottement de Coulomb. Comptes Rendus Mécanique, 2008, 336 (7), pp.606 - 611. 10.1016/j.crme.2008.05.003 . hal-01007406

\section{HAL Id: hal-01007406 \\ https://hal.science/hal-01007406}

Submitted on 22 Oct 2016

HAL is a multi-disciplinary open access archive for the deposit and dissemination of scientific research documents, whether they are published or not. The documents may come from teaching and research institutions in France or abroad, or from public or private research centers.
L'archive ouverte pluridisciplinaire HAL, est destinée au dépôt et à la diffusion de documents scientifiques de niveau recherche, publiés ou non, émanant des établissements d'enseignement et de recherche français ou étrangers, des laboratoires publics ou privés. 


\title{
Une formulation variationnelle du problème de contact avec frottement de Coulomb
}

\author{
Anh Le van, Tai H.T. Nguyen \\ GeM (Institut de recherche en génie civil et mécanique), Faculté des sciences, Université de Nantes, \\ 2, rue de la Houssinière, BP 92208, 44322 Nantes cedex 3, France
}

\begin{abstract}
Dans ce travail, on propose une formulation variationnelle du problème de contact en grandes transformations avec frottement de Coulomb. Il s'agit d'une formulation mixte où les champs inconnus sont à la fois le champ des déplacements et le champ des multiplicateurs, et où les fonctions poids sont le champ des déplacements virtuels et le champ des multiplicateurs virtuels. On montre que le problème faible proposé équivaut bien au problème fort de contact et que les multiplicateurs sont égaux aux contraintes de contact.
\end{abstract}

Mots-clés : Frottement ; Contact unilatéral

\section{A variational formulation for the contact problem with Coulomb friction}

A variational relationship is proposed as the weak form of the large deformation contact problem with Coulomb friction. It is a mixed relationship involving both the displacements and the multipliers; the weighting functions are the virtual displacements and the virtual multipliers. It is shown that the proposed weak form is equivalent to the strong form of the initial/boundary value contact problem and the multipliers are equal to the contact tractions.

Keywords: Friction; Unilateral contact

\section{Formulation forte du problème de contact en grandes transformations}

On travaille dans un référentiel $R_{O}$ défini par un espace affine à trois dimensions représentant l'espace physique et un espace affine à une dimension représentant le temps. Un point origine étant fixé, on confondra l'espace affine ainsi que son espace vectoriel associé à l'espace $\mathbb{R}^{3}$. Un point et son vecteur position seront ainsi confondus à un vecteur à trois composantes de $\mathbb{R}^{3}$. Considérons deux corps en mouvement, repérés par les indices 1 et 2 , de position 
de référence $\Omega_{o}^{(1)}$ et $\Omega_{o}^{(2)}$, respectivement. Le problème mécanique est décrit selon le point de vue de la formulation lagrangienne totale. Sur l'intervalle de temps $[O, T]$ de l'étude, les deux corps subissent les transformations $\boldsymbol{\phi}^{(i)}$ définies par

$$
\forall i \in\{1,2\}, \forall t \in[O, T], \quad \boldsymbol{\phi}^{(i)}(\cdot, t): \Omega_{o}^{(i)} \ni \mathbf{X}^{(i)} \mapsto \mathbf{x}^{(i)}=\boldsymbol{\phi}^{(i)}\left(\mathbf{X}^{(i)}, t\right)
$$

où $\mathbf{X}^{(i)}$ désigne la position de référence d'une particule du corps numéro $i, \mathbf{x}^{(i)}$ la position de la même particule à l'instant courant $t$. Le champ de déplacement dans le corps numéro $i$ est noté $\mathbf{U}^{(i)}$. L'équation locale du mouvement s'écrit

$$
\forall i \in\{1,2\}, \forall \mathbf{X}^{(i)} \in \Omega_{o}^{(i)}, \quad \operatorname{div} \Pi^{(i)}+\rho_{o}^{(i)} \mathbf{f}^{(i)}=\rho_{o}^{(i)} \ddot{\mathbf{U}}^{(i)}
$$

où $\Pi^{(i)}$ est le premier tenseur de contrainte de Piola-Kirchhoff dans le corps numéro $i, \rho_{o}^{(i)}$ la masse volumique et $\mathbf{f}^{(i)}$ la force massique. Pendant leurs mouvements, les corps sont éventuellement en contact sur un certain laps de temps. Pour le corps numéro $i$, on définit une partition de sa frontière $S_{o}^{(i)}$ en trois parties disjointes notées $S_{o U}^{(i)}, S_{o T}^{(i)}$ et $S_{o c}^{(i)}$ telles que $S_{o U}^{(i)}$ et $S_{o T}^{(i)}$ sont des portions de frontière où le déplacement et le vecteur contrainte sont imposés, respectivement, et que $S_{o c}^{(i)}$ est la portion de frontière où le contact a lieu potentiellement avec l'autre corps pour $t \in[O, T]$. Dans certains cas, $S_{o c}^{(i)}$ peut contenir aussi des points où le déplacement est imposé. Les conditions aux limites sur $S_{o U}^{(i)}$ et $S_{o T}^{(i)}$ s'écrivent

$$
\mathbf{U}^{(i)}=\overline{\mathbf{U}}^{(i)} \quad \operatorname{sur} S_{o U}^{(i)}, \quad \Pi^{(i)} \mathbf{N}^{(i)}=\overline{\mathbf{T}}^{(i)} \quad \operatorname{sur} S_{o T}^{(i)}
$$

où $\overline{\mathbf{U}}^{(i)}, \overline{\mathbf{T}}^{(i)}$ désignent respectivement le déplacement imposé et le vecteur contrainte nominale imposée, $\mathbf{N}^{(i)}$ la normale unitaire extérieure au point $\mathbf{X}^{(i)}$ à la surface $S_{o}^{(i)}$. Les conditions aux limites sur les surfaces de contact seront présentées plus loin. Enfin, les conditions initiales du problème s'écrivent

$$
\forall \mathbf{X}^{(i)} \in \Omega_{o}^{(i)}, \quad \mathbf{U}^{(i)}\left(\mathbf{X}^{(i)}, 0\right)=\mathbf{0}, \quad \dot{\mathbf{U}}^{(i)}\left(\mathbf{X}^{(i)}, 0\right)=\overline{\mathbf{V}}_{0}^{(i)}\left(\mathbf{X}^{(i)}\right) \text { (vitesses données) }
$$

Les positions actuelles de $\Omega_{o}^{(i)}, S_{o}^{(i)}, S_{o U}^{(i)}, S_{o T}^{(i)}$ et $S_{o c}^{(i)}$ sont notées $\Omega^{(i)}, S^{(i)}, S_{U}^{(i)}, S_{T}^{(i)}$ et $S_{c}^{(i)}$, respectivement. Ainsi par exemple, $S_{c}^{(i)}=\boldsymbol{\phi}^{(i)}\left(S_{o c}^{(i)}, t\right)$. Pour décrire le mouvement relatif des deux corps, il s'avère commode de choisir l'un des deux corps, disons le corps numéro 2 (cible), comme référence et de décrire la proximité de l'autre, donc le corps numéro 1 (contacteur), par rapport à lui. On suppose que la surface de référence $S_{o c}^{(i)}, i \in\{1,2\}$, est paramétrisée par l'application $\boldsymbol{\theta}^{(i)}$ et qu'à chaque instant $t$, la surface actuelle $S_{c}^{(i)}$ est paramétrisée par l'application $\boldsymbol{\psi}^{(i)}(\cdot, t)=$ $\boldsymbol{\phi}^{(i)}(\cdot, t) \circ \boldsymbol{\theta}^{(i)}$, comme montre le schéma suivant

$$
\begin{aligned}
& \text { un domaine de } \mathbb{R}^{2} \rightarrow S_{o c}^{(i)} \subset \mathbb{R}^{3} \rightarrow S_{c}^{(i)} \subset \mathbb{R}^{3} \\
& \xi^{(i)}=\left(\xi^{(i) 1}, \xi^{(i) 2}\right) \mapsto \mathbf{X}^{(i)}=\boldsymbol{\theta}^{(i)}\left(\xi^{(i)}\right) \mapsto \mathbf{x}^{(i)}=\boldsymbol{\phi}^{(i)}\left(\mathbf{X}^{(i)}, t\right)=\boldsymbol{\psi}^{(i)}\left(\xi^{(i)}, t\right)
\end{aligned}
$$

\subsection{Cinématique du contact}

Considérons un point particulier $\mathbf{X} \in S_{o c}^{(1)}$, dont la position actuelle à l'instant $t$ est $\mathbf{x}=\boldsymbol{\phi}^{(1)}(\mathbf{X}, t) \in S_{c}^{(1)}$. On définit le point $\mathbf{Y} \in S_{o c}^{(2)}$, de position actuelle $\mathbf{y}=\boldsymbol{\phi}^{(2)}(\mathbf{Y}, t) \in S_{c}^{(2)}$, tel que

$$
\mathbf{Y}=\arg \min _{\mathbf{X}^{(2)} \in S_{o c}^{(2)}}\left\|\boldsymbol{\phi}^{(1)}(\mathbf{X}, t)-\boldsymbol{\phi}^{(2)}\left(\mathbf{X}^{(2)}, t\right)\right\|
$$

Ayant associé au point $\mathbf{X}$ (au moins) un point $\mathbf{Y}$, la proximité $g$ est définie par

$$
g=-\boldsymbol{v}\left(\boldsymbol{\phi}^{(1)}(\mathbf{X}, t)-\boldsymbol{\phi}^{(2)}(\mathbf{Y}, t)\right)
$$

où $\boldsymbol{v}$ est la normale au point $\mathbf{y}$. On peut vérifier que $g$ est une grandeur objective. Notons $\mathbf{V}^{(1)}(\mathbf{X}, t)$ la vitesse (à l'instant $t$ et dans le référentiel $R_{o}$ ) de la particule $\mathbf{X}, \mathbf{V}^{(2)}(\mathbf{Y}, t)$ la vitesse (à $t$ et dans $R_{o}$ ) de la particule $\mathbf{Y}$ et $\boldsymbol{\eta}$ l'antécédent de Y. La vitesse relative de contact objective est définie par [1-3]

$$
\mathbf{V}=\mathbf{V}^{(1)}(\mathbf{X}, t)-\mathbf{V}^{(2)}(\mathbf{Y}(\mathbf{X}, t), t)+g \dot{\boldsymbol{v}}=-\dot{g} \boldsymbol{v}+\dot{\eta}^{\alpha} \mathbf{a}_{\alpha}, \quad \mathbf{a}_{\alpha}=\frac{\partial \boldsymbol{\psi}^{(2)}}{\partial \xi^{(2) \alpha}}(\boldsymbol{\eta}(\mathbf{X}, t), t)
$$

A partir de la vitesse (8), on définit la vitesse relative normale $\mathbf{V}_{N}$ et la vitesse de glissement $\mathbf{V}_{T}$ :

$$
\mathbf{V}_{N}=-\dot{g} \boldsymbol{v}, \quad \mathbf{V}_{T}=\dot{\eta}^{\alpha} \mathbf{a}_{\alpha}
$$




\subsection{Sthénique du contact}

Soit $\mathbf{T}(\mathbf{X}, t)=\Pi^{(1)} \cdot \mathbf{N}$ le vecteur contrainte nominale de Piola-Kirchhoff en un point $\mathbf{X} \in S_{o c}^{(1)}$ ( $\mathbf{N}$ est la normale en $\mathbf{X}$ à $\left.S_{o c}^{(1)}\right)$. Il est décomposé dans la base en $\mathbf{y} \in S_{c}^{(2)}$ comme suit

$\mathbf{T}(\mathbf{X}, t)=T_{N}(\mathbf{X}, t) \boldsymbol{v}-\mathbf{T}_{T}(\mathbf{X}, t) \quad \operatorname{avec} \mathbf{T}_{T}=T_{T \alpha} \mathbf{a}^{\alpha}$

où $\mathbf{a}^{\alpha}$ est le vecteur dual de $\mathbf{a}_{\alpha}$. La composante normale $T_{N}$ est la pression nominale de contact. La composante $\mathbf{T}_{T}$ est l'opposé de la contrainte nominale de frottement.

\subsection{Lois de contact}

Les lois de contact sont écrites sous forme mixte où interviennent la vitesse de glissement lagrangienne et les contraintes nominales de contact. La loi de contact normal s'écrit

$$
\forall t, \forall \mathbf{X} \in S_{o c}^{(1)}, \quad g(\mathbf{X}, t) \leqslant 0 \quad \begin{cases}\text { si } g(\mathbf{X}, t)<0, & \text { alors } T_{N}(\mathbf{X}, t)=0 \\ \text { si } g(\mathbf{X}, t)=0, & \text { alors } T_{N}(\mathbf{X}, t) \geqslant 0\end{cases}
$$

Nous utilisons la loi de contact tangentiel de Coulomb, en notant $\mu>0$ le coefficient de frottement :

$\forall t, \forall \mathbf{X} \in S_{o c}^{(1)}$

(a) $g(\mathbf{X}, t)<0 \Rightarrow \mathbf{T}_{T}(\mathbf{X}, t)=\mathbf{0}$, pas de relation $\operatorname{sur} \mathbf{V}_{T}(\mathbf{X}, t)$

(b) $g(\mathbf{X}, t)=0 \Rightarrow\left\|\mathbf{T}_{T}(\mathbf{X}, t)\right\| \leqslant \mu T_{N}(\mathbf{X}, t) \quad \begin{cases}\text {. si }\left\|\mathbf{T}_{T}\right\|<\mu T_{N}, & \mathbf{V}_{T}=\mathbf{0} \\ \text {. si }\left\|\mathbf{T}_{T}\right\|=\mu T_{N}, & \mathbf{V}_{T} \wedge \mathbf{T}_{T}=\mathbf{0} \text { et } \mathbf{V}_{T} \cdot \mathbf{T}_{T} \geqslant 0\end{cases}$

On peut vérifier que les lois de contact normal et tangentiel sont objectives.

\section{Formulation variationnelle du problème de contact}

Dans ce paragraphe, le problème de contact est réécrit sous la forme d'une relation variationnelle mixte qui fait intervenir les champs des déplacements virtuels $\mathbf{U}^{(i) *}\left(\mathbf{X}^{(i)}\right), i \in\{1,2\}$, dépendant du point $\mathbf{X}^{(i)} \in \Omega_{o}^{(i)}$, et les champs de multiplicateurs virtuels $\lambda_{N}^{*}(\mathbf{X})$ et $\lambda_{T}^{*}(\mathbf{X})$ dépendant du point $\mathbf{X} \in S_{o c}^{(1)}$. Ces champs virtuels sont des champs arbitraires supposés suffisamment régulières pour qu'on puisse les intégrer ou les différentier à l'ordre souhaité. Ayant choisi deux scalaires positifs $\epsilon_{N}, \epsilon_{T}$ fixés, l'énoncé de la forme faible du problème est le suivant :

Proposition 1. Les champs solutions du problème fort - à savoir $\mathbf{U}^{(1)}, \mathbf{U}^{(2)}$ dans $\Omega_{o}^{(1)}, \Omega_{o}^{(2)}$ et $T_{N}, \mathbf{T}_{T}, \mathbf{V}_{T}$ sur $S_{o c}^{(1)}-$ vérifient la relation suivante, où $\lambda_{N}=T_{N}$ et $\lambda_{T}=\mathbf{T}_{T}$ :

$$
\begin{aligned}
& \forall t, \forall \mathbf{U}^{(1) *}, \forall \mathbf{U}^{(2) *}, \forall \lambda_{N}^{*}, \forall \lambda_{T}^{*} \\
& \sum_{i=1}^{2}\left\{-\int_{\Omega_{o}^{(i)}} \Pi^{(i) T}: \nabla_{\mathbf{X}^{(i)}} \mathbf{U}^{(i) *} \mathrm{~d} \Omega_{o}+\int_{\Omega_{o}^{(i)}} \rho_{o}^{(i)} \mathbf{f}^{(i)} \mathbf{U}^{(i) *} \mathrm{~d} \Omega_{o}+\int_{S_{o U}^{(i)} \cup S_{o T}^{(i)}} \mathbf{T}^{(i)} \mathbf{U}^{(i) *} \mathrm{~d} S_{o}\right\} \\
& \quad+\int_{S_{o c}^{(1)}}\left[\left\langle\lambda_{N}+\epsilon_{N} g\right\rangle \mathbf{v}-\left(1-\left\langle 1-\frac{\mu\left\langle\lambda_{N}+\epsilon_{N} g\right\rangle}{\left\|\lambda_{T}+\epsilon_{T} \mathbf{V}_{T}\right\|}\right\rangle\right)\left(\lambda_{T}+\epsilon_{T} \mathbf{V}_{T}\right)\right]\left(\mathbf{U}^{(1) *}(\mathbf{X})-\mathbf{U}^{(2) *}(\mathbf{Y}(\mathbf{X}))\right) \mathrm{d} S_{o} \\
& \quad+\int_{S_{o c}^{(1)}}\left\{\left(\lambda_{N}-\left\langle\lambda_{N}+\epsilon_{N} g\right\rangle\right) \frac{\lambda_{N}^{*}}{\epsilon_{N}}+\left[\lambda_{T}-\left(1-\left\langle 1-\frac{\mu\left\langle\lambda_{N}+\epsilon_{N} g\right\rangle}{\left\|\lambda_{T}+\epsilon_{T} \mathbf{V}_{T}\right\|}\right\rangle\right)\left(\lambda_{T}+\epsilon_{T} \mathbf{V}_{T}\right)\right] \frac{\lambda_{T}^{*}}{\epsilon_{T}}\right\} \mathrm{d} S_{o} \\
& =\sum_{i=1}^{2} \int_{\Omega_{o}^{(i)}} \rho_{o}^{(i)} \ddot{\mathbf{U}}^{(i)} \mathbf{U}^{(i) *} \mathrm{~d} \Omega_{o}
\end{aligned}
$$

où $\langle\cdot\rangle$ désigne la fonction "partie positive» $:\langle a\rangle=$ a si $a \geqslant 0,=0$ si a $<0$, et où $\left\langle 1-\frac{\mu\left\langle\lambda_{N}+\epsilon_{N} g\right\rangle}{\left\|\lambda_{T}+\epsilon_{T} \mathbf{V}_{T}\right\|}\right\rangle$ doit être remplacé par 0 en tout point de $S_{\text {oc }}^{(1)}$ où $\lambda_{T}+\epsilon_{T} \mathbf{V}_{T}=\mathbf{0}$. 
Inversement, les surfaces $S_{c}^{(1)}$ et $S_{c}^{(2)}$ étant paramétrisées par (un domaine de $\mathbb{R}^{2}$ ) $\ni \boldsymbol{\xi} \mapsto \mathbf{x} \in S_{c}^{(1)} \subset \mathbb{R}^{3}$ et (un domaine de $\mathbb{R}^{2}$ ) $\ni \boldsymbol{\eta} \mapsto \mathbf{y} \in S_{c}^{(2)} \subset \mathbb{R}^{3}$, supposons qu'il existe une bijection (au moins par morceaux) $S_{c}^{(1)} \ni \mathbf{x} \mapsto$ $\mathbf{y} \in S_{c}^{(2)}$ entre les deux surfaces de contact. Alors, la relation (13) implique à tout instant $t$ les équations locales suivantes :

(1) L'équation locale de la dynamique (2) dans les deux corps 1 et 2.

(2) La relation (3b) sur la portion de frontière $S_{o}^{(i)} \backslash S_{o c}^{(i)}=S_{o T}^{(i)} \cup S_{o U}^{(i)}: \Pi^{(i)} \mathbf{N}^{(i)}=\mathbf{T}^{(i)}$.

(3) Les égalités entre les contraintes nominales et les multiplicateurs sur la surface contactrice $S_{o c}^{(1)}$

$$
\forall \mathbf{X} \in S_{o c}^{(1)}, \quad \mathbf{T}^{(1)}=\boldsymbol{\Pi}^{(1)} \mathbf{N}^{(1)}=\lambda_{N} \boldsymbol{v}-\lambda_{T} \quad \Leftrightarrow \quad T_{N}=\mathbf{T}^{(1)} \boldsymbol{v}=\lambda_{N} \geqslant 0 \quad \text { et } \quad \mathbf{T}_{T}=\lambda_{T}
$$

(4) Les lois de contact normal (11) et tangentiel (12).

(5) La relation suivante qui traduit l'équilibre des contraintes de contact à l'interface de contact

$$
\forall \mathbf{X} \in S_{o c}^{(1)}, \quad \mathbf{T}^{(2)}(\mathbf{Y}(\mathbf{X}, t)) \mathrm{d} S_{o c}^{(2)}=-\left(\lambda_{N} \boldsymbol{v}-\lambda_{T}\right) \frac{\| \mathbf{X}, \xi^{1} \wedge \mathbf{X}, \xi^{2}}{\left\|\mathbf{Y},_{\eta^{1}} \wedge \mathbf{Y},_{\eta^{2}}\right\|} \frac{D \xi}{D \eta} \mathrm{d} S_{o c}^{(2)}=-\mathbf{T}^{(1)}(\mathbf{X}) \mathrm{d} S_{o c}^{(1)}
$$

Démonstration. L'énoncé direct étant facile à vérifier, il suffit de démontrer l'énoncé réciproque.

- Comme (13) est valable quels que soient les champs virtuels $\mathbf{U}^{(1) *}, \mathbf{U}^{(2) *}, \lambda_{N}^{*}$ et $\lambda_{T}^{*}$, prenons $\mathbf{U}^{(1) *}=\mathbf{U}^{(2) *}=\mathbf{0}$ et $\lambda_{T}^{*}=\mathbf{0}$ en gardant $\lambda_{N}^{*}$ quelconque. Ceci conduit à

$$
\lambda_{N}=\left\langle\lambda_{N}+\epsilon_{N} g\right\rangle \quad \operatorname{sur} S_{o c}^{(1)}
$$

dont la première conséquence est que $\lambda_{N} \geqslant 0$. Ensuite, deux cas exclusifs se présentent :

. ou bien $\lambda_{N}+\epsilon_{N} g<0$, alors (16) donne $\lambda_{N}=0$, d'où $g<0 \quad$ (puisque $\lambda_{N}+\epsilon_{N} g<0$ )

. ou bien $\lambda_{N}+\epsilon_{N} g \geqslant 0$, alors (16) donne $g=0$, d'où $\lambda_{N} \geqslant 0 \quad$ (puisque $\lambda_{N}+\epsilon_{N} g \geqslant 0$ )

On peut regrouper les résultats de ces deux cas comme suit :

$$
g \leqslant 0 ; \quad \text { si } g<0, \text { alors } \lambda_{N}=0, \quad \text { si } g=0, \text { alors } \lambda_{N} \geqslant 0
$$

- Reprenons maintenant (13) en y faisant cette fois-ci $\mathbf{U}^{(1) *}=\mathbf{U}^{(2) *}=\mathbf{0}$ et $\lambda_{N}^{*}=0, \lambda_{T}^{*}$ restant quelconque. Le théorème de localisation est applicable puisque le facteur de $\lambda_{T}^{*}$ est continu. Mettons-nous dans un premier temps en un point de $S_{o c}^{(1)}$ où $\lambda_{T}+\epsilon_{T} \mathbf{V}_{T} \neq \mathbf{0}$, on a en ce point

$$
\lambda_{T}=\left(1-\left\langle 1-\frac{\mu\left\langle\lambda_{N}+\epsilon_{N} g\right\rangle}{\left\|\lambda_{T}+\epsilon_{T} \mathbf{V}_{T}\right\|}\right\rangle\right)\left(\lambda_{T}+\epsilon_{T} \mathbf{V}_{T}\right)
$$

dont la première conséquence est que $\mathbf{V}_{T}$ est parallèle à $\lambda_{T}$. On va montrer un résultat plus précis, à savoir que $\mathbf{V}_{T}$ est dirigé dans le même sens que $\lambda_{T}$. Pour cela, notons provisoirement $\alpha=\frac{\mu\left\langle\lambda_{N}+\epsilon_{N} g\right\rangle}{\left\|\lambda_{T}+\epsilon_{T} \mathbf{V}_{T}\right\|} \geqslant 0$. La relation (19) s'écrit alors

$$
\langle 1-\alpha\rangle \lambda_{T}=(1-\langle 1-\alpha\rangle) \epsilon_{T} \mathbf{V}_{T}
$$

Comme $\alpha \geqslant 0$ et $1-\langle 1-\alpha\rangle \geqslant 0, \forall \alpha$, on a les cas exclusifs suivants en fonction de la valeur de $\alpha$ :

. si $\alpha=0$, alors (20) s'écrit $\lambda_{T}=\mathbf{0}$.

. si $0<\alpha<1$, alors $1-\alpha>0$ et $1-\langle 1-\alpha\rangle>0$. L'égalité (20) implique que $(1-\alpha) \lambda_{T}=\alpha \epsilon_{T} \mathbf{V}_{T}$.

. si $\alpha \geqslant 1$, alors (20) donne $\mathbf{V}_{T}=\mathbf{0}$.

Ainsi, dans tous les cas $\mathbf{V}_{T}$ est bien dirigé dans le même sens que $\lambda_{T}$. Distinguons maintenant deux cas :

. si $\lambda_{N}+\epsilon_{N} g<0$, alors on a d'après (17a) et (19) : $g<0$ et $\boldsymbol{\lambda}_{T}=\mathbf{0}$.

. si $\lambda_{N}+\epsilon_{N} g \geqslant 0$, alors on a d'après (17b) $g=0$, et $1-\left\langle 1-\frac{\mu\left\langle\lambda_{N}+\epsilon_{N} g\right\rangle}{\left\|\lambda_{T}+\epsilon_{T} \mathbf{V}_{T}\right\|}\right\rangle=1-\left\langle 1-\frac{\mu\left(\lambda_{N}+\epsilon_{N} g\right)}{\left\|\lambda_{T}+\epsilon_{T} \mathbf{V}_{T}\right\|}\right\rangle$.

Deux sous-cas exclusifs se présentent ici :

- si $\left\|\lambda_{T}+\epsilon_{T} \mathbf{V}_{T}\right\|<\mu\left(\lambda_{N}+\epsilon_{N} g\right)$, alors (19) implique $\mathbf{V}_{T}=\mathbf{0}$. Comme on vient de démontrer que $g=0$, on a $\left\|\lambda_{T}\right\|<\mu \lambda_{N}$.

- si $\left\|\lambda_{T}+\epsilon_{T} \mathbf{V}_{T}\right\| \geqslant \mu\left(\lambda_{N}+\epsilon_{N} g\right)$, alors (19) implique $\lambda_{T}=\mu\left(\lambda_{N}+\epsilon_{N} g\right) \frac{\lambda_{T}+\epsilon_{T} \mathbf{V}_{T}}{\left\|\lambda_{T}+\epsilon_{T} \mathbf{V}_{T}\right\|}$. 
En prenant la norme de la dernière égalité, sachant qu'ici $\lambda_{N}+\epsilon_{N} g \geqslant 0$ et $g=0$, on obtient $\left\|\lambda_{T}\right\|=\mu \lambda_{N}$. On peut regrouper les derniers résultats comme suit :

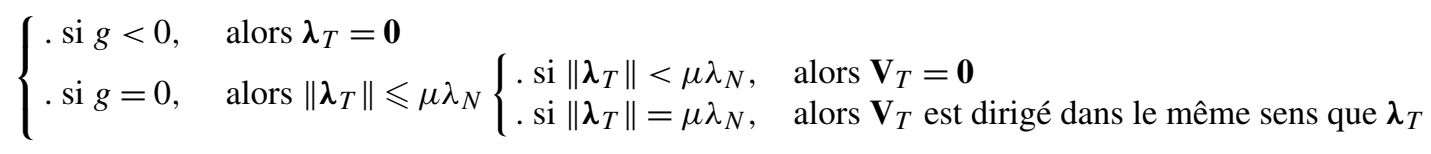

- Ecrivons maintenant (13) en y faisant $\lambda_{N}^{*}=0, \lambda_{T}^{*}=\mathbf{0}$ et y en laissant $\mathbf{U}^{(1) *}$ et $\mathbf{U}^{(2) *}$ quelconques. En appliquant le théorème de la divergence, on arrive à l'égalité suivante

$$
\begin{aligned}
\sum_{i=1}^{2} & \left\{\int_{\Omega_{o}^{(i)}} \mathbf{U}^{(i) *}\left(\operatorname{div} \boldsymbol{\Pi}^{(i)}+\rho_{o}^{(i)} \mathbf{f}^{(i)}\right) \mathrm{d} \Omega_{o}-\int_{S_{o U}^{(i)} \cup S_{o T}^{(i)}} \mathbf{U}^{(i) *} \cdot\left(\boldsymbol{\Pi}^{(i)} \cdot \mathbf{N}^{(i)}-\mathbf{T}^{(i)}\right) \mathrm{d} S_{o}\right\} \\
& \quad-\int_{S_{o c}^{(1)}} \mathbf{U}^{(1) *} \cdot \boldsymbol{\Pi}^{(1)} \cdot \mathbf{N}^{(1)} \mathrm{d} S_{o}+\int_{S_{o c}^{(1)}} \mathbf{U}^{(1) *}\left[\left\langle\lambda_{N}+\epsilon_{N} g\right\rangle \boldsymbol{v}-\left(1-\left\langle 1-\frac{\mu\left\langle\lambda_{N}+\epsilon_{N} g\right\rangle}{\left\|\lambda_{T}+\epsilon_{T} \mathbf{V}_{T}\right\|}\right\rangle\right)\left(\lambda_{T}+\epsilon_{T} \mathbf{V}_{T}\right)\right] \mathrm{d} S_{o} \\
& -\int_{S_{o c}^{(2)}} \mathbf{U}^{(2) *} \cdot \boldsymbol{\Pi}^{(2)} \cdot \mathbf{N}^{(2)} \mathrm{d} S_{o}-\int_{S_{o c}^{(1)}} \mathbf{U}^{(2) *}\left[\left\langle\lambda_{N}+\epsilon_{N} g\right\rangle \mathbf{v}-\left(1-\left\langle 1-\frac{\mu\left\langle\lambda_{N}+\epsilon_{N} g\right\rangle}{\left\|\lambda_{T}+\epsilon_{T} \mathbf{V}_{T}\right\|}\right\rangle\right)\left(\lambda_{T}+\epsilon_{T} \mathbf{V}_{T}\right)\right] \mathrm{d} S_{o} \\
= & \sum_{i=1}^{2} \int_{\Omega_{o}^{(i)}} \rho_{o}^{(i)} \ddot{\mathbf{U}}^{(i)} \mathbf{U}^{(i) *} \mathrm{~d} \Omega_{o}
\end{aligned}
$$

Transformons la dernière intégrale du premier membre étendue sur $S_{o c}^{(1)}$ en une intégrale sur $S_{o c}^{(2)}$, à l'aide la chaîne de bijections $\boldsymbol{\xi} \mapsto \mathbf{X} \mapsto \mathbf{Y} \mapsto \eta$. On a

$$
\mathrm{d} S_{o}^{(1)}=\frac{\left\|\mathbf{X}, \xi^{1} \wedge \mathbf{X}, \xi^{2}\right\|}{\left\|\mathbf{Y},,_{\eta^{1}} \wedge \mathbf{Y},,_{\eta^{2}}\right\|} \frac{D \xi}{D \eta} \mathrm{d} S_{o}^{(2)}
$$

En reportant (23) dans (22), on obtient les relations (2), (3b), (15) et

$$
\mathbf{T}^{(1)}=\boldsymbol{\Pi}^{(1)} \mathbf{N}^{(1)}=\left\langle\lambda_{N}+\epsilon_{N} g\right\rangle \boldsymbol{v}-\left(1-\left\langle 1-\frac{\mu\left\langle\lambda_{N}+\epsilon_{N} g\right\rangle}{\left\|\lambda_{T}+\epsilon_{T} \mathbf{V}_{T}\right\|}\right\rangle\right)\left(\lambda_{T}+\epsilon_{T} \mathbf{V}_{T}\right) \quad \operatorname{sur} S_{o c}^{(1)}
$$

En comparant (24) avec la définition (10) $\mathbf{T}^{(1)}=T_{N} \boldsymbol{v}-\mathbf{T}_{T}$, et en utilisant les relations (16) et (19), on déduit les égalités (14) entre les contraintes et les multiplicateurs. On obtient ensuite les lois de contact normal (11) et tangentiel (12) grâce à (14), (18) et (21).

- Reste à considérer les points de $S_{o c}^{(1)}$ où $\lambda_{T}+\epsilon_{T} \mathbf{V}_{T}=\mathbf{0}$. L'égalité (19) est alors remplacée par $\lambda_{T}=\lambda_{T}+$ $\epsilon_{T} \mathbf{V}_{T}$, ce qui implique $\lambda_{T}=\mathbf{V}_{T}=\mathbf{0}$. La suite du raisonnement est analogue à celle dans le cas $\lambda_{T}+\epsilon_{T} \mathbf{V}_{T} \neq \mathbf{0}$ tout en étant plus simple. On vérifie facilement que la loi de contact tangentiel (12) ainsi que les relations (14) et (15) sont satisfaites aux points où $\lambda_{T}+\epsilon_{T} \mathbf{V}_{T}=\mathbf{0}$. CQFD

\section{Conclusion}

\subsection{Résultat obtenu}

L'équation variationnelle (13) est une généralisation du principe des travaux virtuels, avec prise en compte du contact avec frottement de Coulomb entre deux corps subissant de grandes transformations. Elle fait intervenir à la fois les déplacements et les multiplicateurs dans une intégrale de surface étendue sur la surface de contact de référence du contacteur. Elle est valable quelles que soient les lois de comportement des deux corps en contact. 


\subsection{Comparaison avec les résultats de la littérature}

Depuis plus de trois décennies, de nombreuses formulations et méthodes de résolution numérique ont été proposées dans la littérature en vue de traiter différents types de problèmes de contact, allant du contact sans frottement en élastostatique linéaire jusqu'au contact frottant en grandes transformations avec des lois d'interface complexes, en statique comme en dynamique. Les principales méthodes utilisées pour inclure les inégalités de contact sont la méthode du multiplicateur de Lagrange, la méthode de pénalité et la méthode du lagrangien augmenté qui combine les avantages des deux premières. Comme la formulation proposée dans ce travail est proche des méthodes de type du lagrangien augmenté, nous allons la comparer à ces dernières seulement.

Le problème de contact frottant en petites transformations a été résolu à l'aide de la méthode du lagrangien augmenté dans [4] et [5]. Le contact sans frottement en grandes transformations a été aussi traité avec succès à l'aide de la même méthode, assez tôt dans [6] où on considère un obstacle plan rigide, puis dans [7] pour le contact entre deux corps élastiques et plus tard dans [8]. Enfin, différentes formulations du problème du contact frottant en grandes transformations entre deux corps déformables ont été données dans [3,9] et [10]. Tous les précédents travaux considéraient le modèle de frottement de Coulomb ou une de ses extensions incluant par exemple des effets micro-mécaniques du contact. Par ailleurs, lors de la résolution numérique les contraintes tangentielles de contact sont toujours (sauf dans $[3,4,7]$ ) calculées par intégration locale des lois de contact au niveau de l'interface et la matrice tangente de contact en est déduite de manière cohérente, selon un procédé analogue au schéma de retour radial en élastoplasticité.

(a) La formulation présentée dans ce travail a un point commun avec les travaux de Curnier et de ses collaborateurs $[3,4,7]$ dont elle s'est inspirée : la loi de contact tangentiel est directement incorporée dans la fonctionnelle (13) du problème si bien qu'elle est automatiquement satisfaite lors de la résolution numérique, sans avoir besoin de recourir à l'intégration locale de la loi de frottement.

(b) Notre formulation diffère cependant de [3,4,7] dans la relation variationnelle (13) et l'égalité (15) obtenue comme une conséquence de (13). Du point de vue pratique, l'expression explicite (13) se prête plus facilement à la discrétisation par éléments finis. Les détails sur le système discret d'équations qui en résulte et sur la matrice tangente de contact correspondante dépassent le cadre de ce papier et ne seront pas fournis ici.

La formulation proposée diffère de $[5,9,10]$ sur les deux points (a) et (b).

\section{Remerciements}

Les auteurs remercient Nguyen Quoc Son, du Laboratoire de mécanique des solides, École polytechnique, pour ses remarques instructives qui ont permis d'affiner la Proposition 1 et d'améliorer la version révisée.

\section{Références}

[1] A. Curnier, Qi.-Ch. He, A. Klarbring, Continuum mechanics modelling of large deformation contact with friction, in: M. Raous, M. Jean, J.J. Moreau (Eds.), Contact Mechanics, Plenum, New York, 1995, pp. 145-158.

[2] A. Klarbring, Large displacement frictional contact: a continuum framework for finite element discretisation, European Journal of Mechanics A/Solids 14 (1995) 237-253.

[3] G. Pietrzak, A. Curnier, Large deformation frictional contact mechanics: continuum formulation and augmented Lagrangian treatment, Computer Methods in Applied Mechanics and Engineering 177 (1999) 351-381.

[4] P. Alart, A. Curnier, A mixed formulation for frictional contact problems prone to Newton like solution methods, Computer Methods in Applied Mechanics and Engineering 92 (1991) 353-375.

[5] G. Zavarise, P. Wriggers, B.A. Schrefler, On augmented Lagrangian algorithms for thermomechanical contact problems with friction, International Journal for Numerical Methods in Engineering 38 (1995) 2929-2949.

[6] R. Glowinski, P. Le Tallec, Augmented Lagrangian and Operator-Splitting Methods in Nonlinear Mechanics, Studies in Applied Mathematics, SIAM, 1989.

[7] J.-H. Heegaard, A. Curnier, An augmented Lagrangian method for discrete large-slip contact problems, International Journal for Numerical Methods in Engineering 36 (1993) 569-593.

[8] G. Zavarise, P. Wriggers, A superlinear convergent augmented Lagrangian procedure for contact problems, Engineering Computations 16 (1999) 88-119.

[9] J.C. Simo, T.A. Laursen, An augmented Lagrangian treatment of contact problems involving friction, Computers and Structures 42 (1992) 97-116.

[10] P. Wriggers, Finite element methods for contact problems with friction, Tribology International 29 (1996) 651-658. 